\title{
Crossroad of infection and autoimmunity in acute liver failure: a case report
}

\author{
Akash Roy ${ }^{1}$, Sunil Taneja ${ }^{*}$, Arka De$^{1}$, Ashim Das $^{2}$, Ajay K. Duseja ${ }^{1}$ and Virendra Singh ${ }^{1}$
}

\begin{abstract}
Background: Acute liver failure (ALF) is a syndromic diagnosis, consisting of jaundice, coagulopathy, and any degree of encephalopathy in a patient without pre-existing liver disease within 26 weeks of the onset of symptoms. Autoimmune hepatitis has a wide range of presentations and can rarely present as ALF, which frequently tends to be autoantibody negative. Tropical infections like dengue, malaria, and leptospirosis, which present as mimickers of ALF, always remain a differential diagnosis of ALF and mandate an etiology specific management. In rare cases, such infections themselves act as a trigger for autoimmunity. We report a case of diagnostic crossroads of infection and autoimmunity, presenting as acute liver failure and describe the challenges in management.
\end{abstract}

Case presentation: A 25-year-old female presented with a syndromic diagnosis of acute liver failure with possibility of tropical illness-related ALF mimic on account of positive Leptospira serology. After initial improvement, there was a rebound worsening of liver functions which prompted a liver biopsy. Biopsy narrowed the differential to Leptospira-associated hepatitis and severe auto-immune hepatitis. Trial of low dose steroid was given which led to dramatic improvement.

Conclusion: Tropical infections can present as ALF mimics and can themselves act as triggers for autoimmunity. Distinguishing such cases from de-novo acute severe autoimmune hepatitis is difficult and requires therapeutic brinksmanship. An early trial of steroid is mandated but comes with its own challenges.

Keywords: Leptospira, Autoimmune hepatitis, Acute liver failure; Case report

\section{Background}

Acute liver failure (ALF) is a syndromic diagnosis characterized acute onset ( $<4$ weeks) of jaundice, coagulopathy, and encephalopathy in a patient without pre-existing liver disease. Tropical infections like dengue, malaria, enteric fever, and leptospirosis often present as mimickers of ALF $[1,2]$. Another etiology of ALF is autoimmune hepatitis, which differs from its classical presentation in being frequently seronegative in such cases [3]. Infections have been associated with $\mathrm{AIH}$ and can serve as a trigger for autoimmunity, thus leading to a grey zone in diagnosis. We report a case of ALF with a diagnostic crossroads between leptospirosis and autoimmunity

\footnotetext{
* Correspondence: drsuniltaneja@hotmail.com

${ }^{1}$ Department of Hepatology, Post Graduate Institute of Medical Education and Research (PGIMER), Chandigarh, India

Full list of author information is available at the end of the article
}

\section{Case presentation}

A 25-year-old female presented with complaints of fever for 2 weeks, jaundice for 1 week, and altered sensorium for 2 days. There was no history of headache, trauma, vision abnormalities, retro-orbital pain, conjunctival suffusion, rashes, or seizures. On examination, she was febrile, normotensive, maintained oxygen saturation, pale, icteric, and had grade II hepatic encephalopathy. Per abdominal examination was unremarkable. There was no significant history of any previous medical comorbidities. Her investigations revealed presence of anemia, leucocytosis deranged liver functions and coagulopathy. On etiological workup, viral markers including IgM hepatitis A, IgM hepatitis E, HBsAg, and anti-HCV were negative, while the IgM antibody for leptospirosis came out to be positive (titers 27.7 NTU). There was no definite history of potential exposure to Leptosira. 
Abdominal ultrasonography showed a normal liver with a span of $12 \mathrm{~cm}$ and a normal spleen, with no free fluid. A syndromic diagnosis of acute liver failure (ALF) with the possibility of Leptospira associated ALF-mimicker was kept. She was managed as per standard ALF protocol along with intravenous doxycycline in view of positive Leptospira serology. The fever subsided, and hepatic encephalopathy improved; however, as liver functions and coagulogram failed to improve, she was given five sessions of plasmapheresis. However, 5 days after plasmapheresis, there was a worsening of liver functions and coagulation [Bilirubin (Total/direct) 35 $\mathrm{mg} / \mathrm{dl} / 24 \mathrm{mg} / \mathrm{dl}$, AST/ALT/ALP 734/656/128 U/L, INR 1.9). The trend in the biochemical parameters is shown in Table 1. The further etiological workup including autoimmune (ANA, SMA, AMA, LKM, PCA ANCA, SLA), total immunoglobulin $G$ levels, viral serologies for cytomegalovirus, Epstein-Barr virus, herpes simplex virus, and Wilson's disease workup were all negative. A transjugular liver biopsy was done, which showed centrizonal necrosis with lymphohistiocytic cell infiltrate with canalicular and intracellular cholestasis with multiple foci of lobular inflammation. The portal tracts showed minimal irregular portal tract expansion, minimal portal tract inflammation, and minimal interface hepatitis (Fig. 1). A histological possibility of Leptospira-associated-acute hepatitis versus acute severe autoimmune hepatitis was kept. She was started on low dose prednisolone ( $20 \mathrm{mg} /$ day), which led to a gradual improvement of liver functions. Subsequently, the steroids were tapered after normalization of LFTs, and presently she is on $5 \mathrm{mg}$ of prednisolone on regular follow.

\section{Discussion}

Tropical febrile syndromes mimicking as ALF is a frequently encountered challenge. The most common etiologies are falciparum malaria, dengue, Leptospirosis, and rickettsial fevers [1]. Identification and differentiation of these specific etiologies is essential to ensure a specific therapy. Features like high-grade fever, persistent febrile spikes after the onset of jaundice, overt bleeding manifestations, and splenomegaly serve as possible indicators of a tropical illness-related ALF. Similarly, laboratory indices like higher AST or LDH than ALT and INR < 1.5, thrombocytopenia, and early-onset hemodynamic instability and renal failure is more common in ALF mimickers $[1,2]$. The clinical indicators of an initial suspicion of Leptospira-associated ALF in our case were persistent febrile spikes and positive serological tests. However, features like an absence of renal dysfunction, lack of hemorrhagic manifestations, and markedly elevated liver enzymes questioned the possibility of a Leptospira related ALF.

After an initial improvement post five sessions of plasmapheresis, there was enzyme elevation and worsening liver function. A detailed workup of possible etiology of acute liver failure was however inconclusive. At this crossroads, the patient was taken up for a transjugular liver biopsy, which was suggestive of acute hepatitis with a diagnostic possibility of Leptospira-associated-acute hepatitis versus acute autoimmune hepatitis. Acute severe AIH (AS-AIH) is an acute presentation ( $\leq 26$ weeks) with an INR of $\geq$ 1.5 without any histological evidence of cirrhosis [3]. It is of importance to note that patients with an acute severe $\mathrm{AIH}$ frequently tend to be seronegative for

Table 1 Hematological and biochemical parameters

\begin{tabular}{|c|c|c|c|c|}
\hline Investigations & Index presentation & Pre-plasmapharesis & Post-plasmapharesis & Post-steroids \\
\hline Hemoglobin (g/dl) & 8.9 & 8.3 & 8.1 & 8.6 \\
\hline Total leucocyte count $\left(/ \mathrm{mm}^{3}\right)$ & 11900 & 10200 & 7300 & 10200 \\
\hline Platelet count $\left(/ \mathrm{mm}^{3}\right)$ & 290000 & 275000 & 184000 & 204000 \\
\hline International normalized ratio (INR) & 2.8 & 2 & 1.3 & 1.1 \\
\hline \multicolumn{5}{|l|}{ Liver function tests (LFT) } \\
\hline - Bilirubin (mg/dl) & 22 & 51 & 19 & 3 \\
\hline • AST (IU) & 1161 & 530 & 212 & 45 \\
\hline • ALT (IU) & 913 & 127 & 239 & 44 \\
\hline - Protein (g/dl) & 6.6 & 6.8 & 6.9 & 7 \\
\hline - Albumin(g/dl) & 3.4 & 3.3 & 3.2 & 3.3 \\
\hline • SAP (IU) & 109 & 127 & 120 & 97 \\
\hline \multicolumn{5}{|l|}{ Renal function tests } \\
\hline - Urea $(\mathrm{mmol} / \mathrm{L})$ & 28 & 31 & 28 & 21 \\
\hline - Creatinine(mg/dl) & 1.0 & 1.1 & 0.9 & 0.9 \\
\hline
\end{tabular}



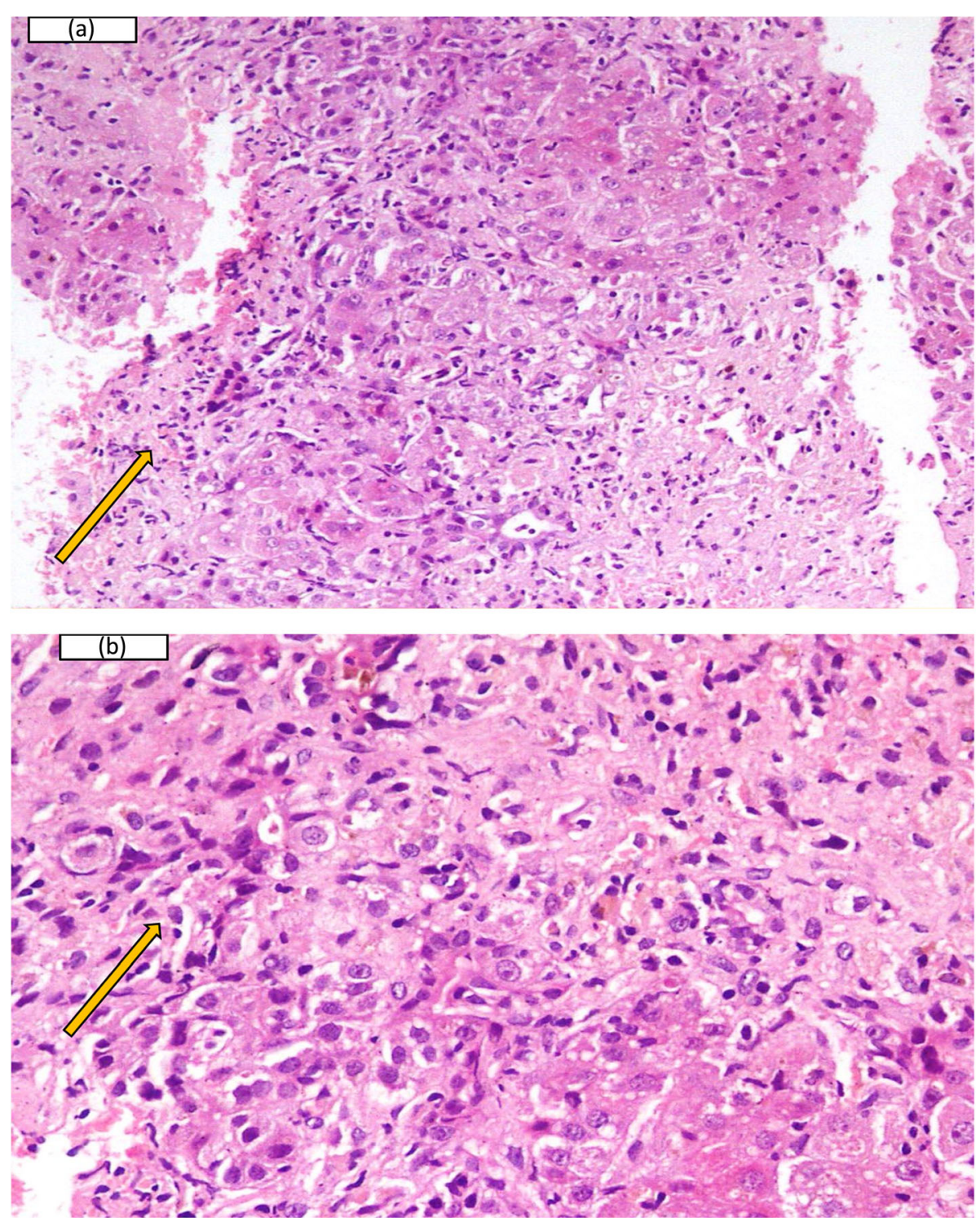

Fig. 1 a H\& E stain showing extensive centrizonal necrosis. b H\&E stain showing necrotic hepatocytes surrounded by lymphohistiocytic infiltrate

autoimmune markers. ANA antibodies are negative or weakly positive in $29-39 \%$ of patients whereas gamma-globulin levels may be normal in $25-39 \%$ thus adding to the diagnostic challenge $[4,5]$. Our patient was started on a steroid trial, to which she responded, although literature suggests that such patients frequently tend to be steroid non-responders and end up requiring liver tranplantation [6].

The two primary differentials in our case were tropical illness-related ALF and AS-AIH. While the diagnosis of AS-AIH itself becomes a challenge due to frequent seronegativity, the other possibility of an infection itself triggering an autoimmune phenomenon adds to the diagnostic puzzle. Pathogens like HAV, HCV, EBV, CMV, HIV, and Leishmania have been postulated to trigger autoimmune hepatitis. However, the literature with Leptsopira as a trigger of autoimmune hepatitis presenting as ALF is scarce with only a single case report in a pediatric patient $[7,8]$. Thus, management of such cases entails an element of therapeutic brinksmanship to closely balance the two contrasting entities of infection and autoimmunity.

\section{Conclusion}

Tropical infections may present as ALF mimics and can themselves act as triggers for autoimmunity. Distinguishing such cases from de-novo acute severe autoimmune hepatitis is difficult and requires therapeutic brinksmanship. An early trial of steroid is mandated in such cases and can alter the course of the disease. 


\section{Key learning}

- Tropical infections can present as ALF mimics

- Clinical pointers and biochemical signatures should be carefully looked for to determine the etiology of an ALF mimicker

- Acute severe AIH can be seronegative in up to onethird of the cases

- Infections can serve as a trigger for autoimmunity and the interplay needs further understanding

- Acute severe AIH with early grades of HE may benefit from a trial of steroids

\begin{abstract}
Abbreviations
AlH: Autoimmune hepatitis; ALF: Acute liver failure; HAV: Hepatitis A virus; HBV: Hepatitis B virus; HCV: Hepatitis C virus; EBV: Epstein-Barr virus; HIV: Human immunodeficiency virus; ANA: Antinuclear antibodies; AMA: Antimitochondrial antibodies; AST: Aspartate transaminase; ALT: Alanine transaminase; ALP: Alkaline phosphatase; LKM: Liver kidney microsome antibodies; PCA: Parietal cell antibodies; SAP: Serum alkaline phosphatase; SMA: Smooth muscles antibodies; SLA: Soluble liver antigen antibodies; INR: International normalized ratio
\end{abstract}

\section{Acknowledgements}

$\mathrm{Ni}$

\section{Authors' contributions}

AR: data compilation and writing; ST: critical revision; AD: critical revision; AD: histopathological support; AKD critical revision; VS: critical revision. All authors have read and approved the manuscript.

\section{Funding}

$\mathrm{Ni}$

Availability of data and materials

Yes

Ethics approval and consent to participate

Written informed consent was obtained from patient.

\section{Consent for publication}

Written informed consent was obtained from patient.

\section{Competing interests}

The authors declare that they have no competing interests.

\section{Author details}

${ }^{1}$ Department of Hepatology, Post Graduate Institute of Medical Education and Research (PGIMER), Chandigarh, India. Department of Pathology, Post Graduate Institute of Medical Education and Research (PGIMER), Chandigarh, India.

Received: 14 October 2020 Accepted: 9 February 2021

Published online: 02 April 2021

\section{References}

1. Patel ND, Amrapurkar D (2006) Differential diagnosis of acute liver failure in India. Ann Hepatol 5(3):150-156

2. Murthy GL, Sahay RK, Sreenivas DV, Sundaram C, Shantaram V (1998) Hepatitis in falciparum malaria. Trop Gastroenterol 19:152-154

3. Czaja AJ (2013) Acute and acute severe (fulminant) autoimmune hepatitis Dig Dis Sci 58:897-914

4. Yasui S, Fujiwara K, Yonemitsu Y, Oda S, Nakano M, Yokosuka O (2011) Clinicopathological features of severe and fulminant forms of autoimmune hepatitis. J Gastroenterol 46:378-390
5. Fujiwara K, Fukuda Y, Yokosuka O (2008) Precise histological evaluation of liver biopsy specimen is indispensable for diagnosis and treatment of acuteonset autoimmune hepatitis. J Gastroenterol 43:951-958

6. Anand L, Choudhury A, Bihari C, Sharma BC, Kumar M, Maiwall R et al (2018) Flare of autoimmune hepatitis causing acute on chronic liver failure: diagnosis and response to corticosteroid therapy. Hepatology 70(2):587-596

7. Christen U, Hintermann E (2019) (2019). Pathogens and autoimmune hepatitis. Clin Exp Immunol 195(1):35-51

8. Urganci N, Kalyoncu D, Cayonu N, Erdem E, Yildirmak Y, Yilmaz B (2011) Acute liver failure, autoimmune hepatitis, and Leptospirosis: a case report. Pediatr Emerg Care 27(10):963-965

\section{Publisher's Note}

Springer Nature remains neutral with regard to jurisdictional claims in published maps and institutional affiliations.

\section{Submit your manuscript to a SpringerOpen ${ }^{\circ}$ journal and benefit from:}

- Convenient online submission

- Rigorous peer review

- Open access: articles freely available online

- High visibility within the field

- Retaining the copyright to your article

Submit your next manuscript at $>$ springeropen.com 